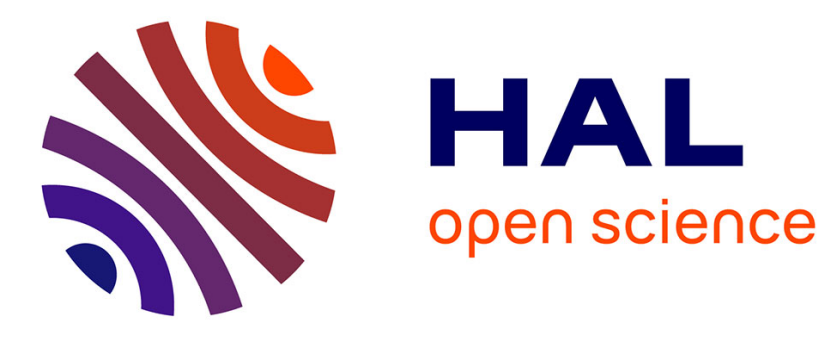

\title{
The Gradient Discretisation Method for Two-phase Discrete Fracture Matrix Models in Deformable Porous Media
}

Francesco Bonaldi, Konstantin Brenner, Jerome Droniou, Roland Masson

\section{- To cite this version:}

Francesco Bonaldi, Konstantin Brenner, Jerome Droniou, Roland Masson. The Gradient Discretisation Method for Two-phase Discrete Fracture Matrix Models in Deformable Porous Media. FVCA 2020 - 9th Conference on Finite Volumes for Complex Applications, Jun 2020, Bergen, Norway. 10.1007/978-3-030-43651-3_26. hal-02454360v3

\section{HAL Id: hal-02454360 \\ https://hal.science/hal-02454360v3}

Submitted on 11 Mar 2020

HAL is a multi-disciplinary open access archive for the deposit and dissemination of scientific research documents, whether they are published or not. The documents may come from teaching and research institutions in France or abroad, or from public or private research centers.
L'archive ouverte pluridisciplinaire $\mathbf{H A L}$, est destinée au dépôt et à la diffusion de documents scientifiques de niveau recherche, publiés ou non, émanant des établissements d'enseignement et de recherche français ou étrangers, des laboratoires publics ou privés. 


\title{
The Gradient Discretisation Method for Two-phase Discrete Fracture Matrix Models in Deformable Porous Media
}

\author{
F. Bonaldi, K. Brenner, J. Droniou, R. Masson
}

\begin{abstract}
We consider a two-phase Darcy flow in a fractured porous medium consisting in a matrix flow coupled with a tangential flow in the fractures, described as a network of planar surfaces. This flow model is also coupled with the mechanical deformation of the matrix assuming that the fractures are open and filled by the fluids, as well as small deformations and a linear elastic constitutive law. The model is discretized using the gradient discretization method [3, which covers a large class of conforming and non conforming discretizations. This framework allows a generic convergence analysis of the coupled model using a combination of discrete functional tools. Here, we describe the model together with its numerical discretisation, and we state the convergence result, whose proof will be detailed in a forthcoming paper. This is, to our knowledge, the first convergence result for this type of models taking into account two-phase flows and the nonlinear poro-mechanical coupling. Previous related works consider a linear approximation obtained for a single phase flow by freezing the fracture conductivity [4].
\end{abstract}

Key words: poromechanics, discrete fracture matrix models, two-phase Darcy flows, Gradient Discretization, convergence analysis

MSC (2010): 65M12, 76S05, 74B10

\section{Continuous model}

We consider a bounded polytopal domain $\Omega$ of $\mathbb{R}^{d}, d \in\{2,3\}$, partitioned into a fracture domain $\Gamma$ and a matrix domain $\Omega \backslash \bar{\Gamma}$. The network of fractures is $\Gamma=$ $\bigcup_{i \in I} \Gamma_{i}$, where each $\Gamma_{i}$ is planar and has therefore two sides denoted by \pm in the

Francesco Bonaldi

Université Côte d'Azur, Inria, CNRS, Laboratoire J.A. Dieudonné, team Coffee, France, e-mail: francesco.bonaldieuniv-cotedazur.fr

Konstantin Brenner

Université Côte d'Azur, Inria, CNRS, Laboratoire J.A. Dieudonné, team Coffee, France, e-mail: konstantin.brenner@univ-cotedazur.fr

Jérôme Droniou

School of Mathematics, Monash University, Victoria 3800, Australia,

e-mail: jerome.droniou@monash.edu

Roland Masson

Université Côte d'Azur, Inria, CNRS, Laboratoire J.A. Dieudonné, team Coffee, France, e-mail: roland.massoneuniv-cotedazur.fr 
matrix domain, with unit normal vectors $\mathbf{n}^{ \pm}$oriented outward to the sides \pm . We denote by $\gamma$ the trace operator on $\Gamma$ for functions in $H^{1}(\Omega)$ and by $\llbracket \cdot \rrbracket$ the normal trace jump operator on $\Gamma$ for functions in $H_{\text {div }}(\Omega \backslash \bar{\Gamma})$.

We denote by $\nabla_{\tau}$ the tangential gradient and by $\operatorname{div}_{\tau}$ the tangential divergence on the fracture network $\Gamma$. The symmetric gradient operator $\mathbb{E}$ is defined such that $\mathbb{C}(\mathbf{v})=\frac{1}{2}\left(\nabla \mathbf{v}+{ }^{t}(\nabla \mathbf{v})\right)$ for a given vector field $\mathbf{v}$.

Let us fix a continuous function $d_{0}: \Gamma \rightarrow(0,+\infty)$ with zero limits at $\partial \Gamma \backslash(\partial \Gamma \cap$ $\partial \Omega$ ) (i.e. the tips of $\Gamma$ ) and stricly positive limits at $\partial \Gamma \cap \partial \Omega$.

Let us introduce the following function spaces: $\mathbf{U}_{0}=\left\{\overline{\mathbf{v}} \in\left(H^{1}(\Omega \backslash \bar{\Gamma})\right)^{d} \mid \gamma_{\partial \Omega} \overline{\mathbf{v}}=\right.$ 0 on $\partial \Omega\}$ for the displacement vector, and $V_{0}=\left\{\bar{v} \in H_{0}^{1}(\Omega) \mid \gamma \bar{v} \in H_{d_{0}}^{1}(\Gamma)\right\}$ for each phase pressure, where the space $H_{d_{0}}^{1}(\Gamma)$ is made of functions $v_{\Gamma}$ in $L^{2}(\Gamma)$, such that $d_{0}^{3 / 2} \nabla_{\tau} v_{\Gamma}$ is in $L^{2}(\Gamma)$, whose traces are continuous at fracture intersections $\partial \Gamma_{i} \cap \partial \Gamma_{j}$ and vanish on the boundary $\partial \Gamma \cap \partial \Omega$.

The matrix and fracture rock types are denoted by the indices $\mathrm{rt}=m$ and $\mathrm{rt}=f$, respectively, and the non-wetting and wetting phases by the superscripts $\alpha=n$ w and $\alpha=\mathrm{w}$, respectively.

Fig. 1 Example of a 2D domain $\Omega$ with its fracture network $\Gamma$, the unit normal vectors $\mathbf{n}^{ \pm}$at $\Gamma$, the phase pressures $\bar{p}^{\alpha}$ in the matrix and $\gamma \bar{p}^{\alpha}$ in the fracture network, the displacement vector field $\overline{\mathbf{u}}$, the matrix Darcy velocities $\mathbf{q}_{m}^{\alpha}$ and the fracture tangential Darcy velocities $\mathbf{q}_{f}^{\alpha}$ integrated along the fracture.

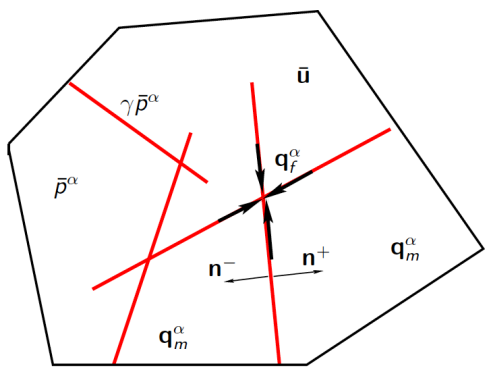

The PDEs model reads: find the phase pressures $\bar{p}^{\alpha}, \alpha \in\{\mathrm{nw}, \mathrm{w}\}$, and the displacement vector field $\overline{\mathbf{u}}$, such that $\bar{p}_{c}=\bar{p}^{\mathrm{nw}}-\bar{p}^{\mathrm{w}}$ and, for $\alpha \in\{\mathrm{nw}, \mathrm{w}\}$,

$$
\begin{cases}\partial_{t}\left(\bar{\phi}_{m} S_{m}^{\alpha}\left(\bar{p}_{c}\right)\right)+\operatorname{div}\left(\mathbf{q}_{m}^{\alpha}\right)=h_{m}^{\alpha} & \text { on }(0, T) \times \Omega \backslash \bar{\Gamma}, \\ \mathbf{q}_{m}^{\alpha}=-\eta_{m}^{\alpha}\left(S_{m}^{\alpha}\left(\bar{p}_{c}\right)\right) \mathbb{K}_{m} \nabla \bar{p}^{\alpha} & \text { on }(0, T) \times \Omega \backslash \bar{\Gamma}, \\ \partial_{t}\left(\bar{d}_{f} S_{f}^{\alpha}\left(\gamma \bar{p}_{c}\right)\right)+\operatorname{div}_{\tau}\left(\mathbf{q}_{f}^{\alpha}\right)-\llbracket \mathbf{q}_{m}^{\alpha} \rrbracket=h_{f}^{\alpha} & \text { on }(0, T) \times \Gamma, \\ \mathbf{q}_{f}^{\alpha}=-\eta_{f}^{\alpha}\left(S_{f}^{\alpha}\left(\gamma \bar{p}_{c}\right)\right)\left(\frac{1}{12} \bar{d}_{f}^{3}\right) \nabla_{\tau} \gamma \bar{p}^{\alpha} & \text { on }(0, T) \times \Gamma, \\ -\operatorname{div}\left(\sigma(\overline{\mathbf{u}})-b \bar{p}_{m}^{E} \mathbb{I}\right)=\mathbf{f} & \text { on }(0, T) \times \Omega \backslash \bar{\Gamma} \\ \sigma(\overline{\mathbf{u}})=2 \mu \mathbb{e}(\overline{\mathbf{u}})+\lambda \operatorname{div}(\overline{\mathbf{u}}) \mathbb{I} & \text { on }(0, T) \times \Omega \backslash \bar{\Gamma},\end{cases}
$$

with

$$
\begin{cases}\partial_{t} \bar{\phi}_{m}=b \operatorname{div} \partial_{t} \overline{\mathbf{u}}+\frac{1}{M} \partial_{t} \bar{p}_{m}^{E} & \text { on }(0, T) \times \Omega \backslash \bar{\Gamma}, \\ \left(\sigma(\overline{\mathbf{u}})-b \bar{p}_{m}^{E} \mathbb{I}\right) \mathbf{n}^{ \pm}=-\bar{p}_{f}^{E} \mathbf{n}^{ \pm} & \text {on }(0, T) \times \Gamma, \\ \bar{d}_{f}=-\llbracket \overline{\mathbf{u}} \rrbracket & \text { on }(0, T) \times \Gamma,\end{cases}
$$

and the initial conditions

$$
\left.\bar{p}^{\alpha}\right|_{t=0}=\bar{p}_{0}^{\alpha},\left.\quad \bar{\phi}_{m}\right|_{t=0}=\bar{\phi}_{m}^{0} .
$$

Here, the equivalent pressures $p_{m}^{E}$ and $p_{f}^{E}$ are defined, following [2], by

$$
\bar{p}_{m}^{E}=\sum_{\alpha \in\{\mathrm{nw}, \mathrm{w}\}} \bar{p}^{\alpha} S_{m}^{\alpha}\left(\bar{p}_{c}\right)-U_{m}\left(\bar{p}_{c}\right), \quad \bar{p}_{f}^{E}=\sum_{\alpha \in\{\mathrm{nw}, \mathrm{w}\}} \gamma \bar{p}^{\alpha} S_{f}^{\alpha}\left(\gamma \bar{p}_{c}\right)-U_{f}\left(\gamma \bar{p}_{c}\right),
$$


where $U_{\mathrm{rt}}\left(\bar{p}_{c}\right)=\int_{0}^{\bar{p}_{c}} q\left(S_{\mathrm{rt}}^{\mathrm{nw}}\right)^{\prime}(q) d q$ is the capillary energy density function for each rock type rt $\in\{m, f\}$. This is a key choice to obtain the energy estimates which are the starting point for the convergence analysis.

We make the following main assumptions on the data:

- For each phase $\alpha \in\{\mathrm{nw}, \mathrm{w}\}$ and rock type $\mathrm{rt} \in\{m, f\}$, the mobility function $\eta_{\mathrm{rt}}^{\alpha}$ is continuous non-decreasing and there exist $0<\eta_{\mathrm{rt}, \min }^{\alpha} \leqslant \eta_{\mathrm{rt}, \max }^{\alpha}<+\infty$ such that $\eta_{\mathrm{rt}, \min }^{\alpha} \leqslant \eta_{\mathrm{rt}}^{\alpha}(s) \leqslant \eta_{\mathrm{rt}, \max }^{\alpha}$ for all $s \in[0,1]$.

- For each rock type $\mathrm{rt} \in\{m, f\}, S_{\mathrm{rt}}^{\mathrm{nw}}$ is a non-decreasing Lipschitz continuous function with values in $[0,1]$, and $S_{\mathrm{rt}}^{\mathrm{w}}=1-S_{\mathrm{rt}}^{\mathrm{nw}}$.

- $b \in[0,1]$ is the Biot coefficient, $M>0$ is the Biot modulus, and $\lambda>0, \mu>0$ are the Lamé coefficients. These coefficients are assumed to be constant for simplicity.

- There exist $0<\phi_{m, \text { min }}^{0} \leqslant \phi_{m, \text { max }}^{0}<1$ such that $\phi_{m, \text { min }}^{0} \leqslant \bar{\phi}_{m}^{0}(\mathbf{x}) \leqslant \phi_{m, \text { max }}^{0}$ for a.e. $\mathbf{x} \in \Omega$.

- The initial fracture aperture satisfies $\bar{d}_{f}^{0}(t, \mathbf{x}) \geqslant d_{0}(\mathbf{x})$ for a.e. $(t, \mathbf{x}) \in(0, T) \times \Gamma$.

- The permeability tensor $\mathbb{K}_{m}$ is symmetric and uniformly elliptic on $\Omega$.

Definition 1 (Weak solution of the model). A weak solution of the model for $\mathbf{f} \in L^{2}(\Omega)^{d}, h_{m}^{\alpha} \in L^{2}((0, T) \times \Omega)$, and $h_{f}^{\alpha} \in L^{2}((0, T) \times \Gamma)$, is given by $\bar{p}^{\alpha} \in$ $L^{2}\left(0, T ; V_{0}\right), \alpha \in\{\mathrm{nw}, \mathrm{w}\}$, and $\overline{\mathbf{u}} \in L^{\infty}\left(0, T ; \mathbf{U}_{0}\right)$, such that for any $\alpha \in\{\mathrm{nw}, \mathrm{w}\}$, $\left.\bar{d}_{f}^{3 / 2} \nabla_{\tau} \gamma \bar{p}^{\alpha} \in L^{2}((0, T) \times \Gamma)\right)^{d}$ and, for all $\bar{\varphi}^{\alpha} \in C_{c}^{\infty}([0, T) \times \Omega)$ and all smooth functions $\overline{\mathbf{v}}:[0, T] \times(\Omega \backslash \bar{\Gamma}) \rightarrow \mathbb{R}^{d}$ vanishing on $\partial \Omega$ and having finite limits on each side of $\Gamma$,

$$
\begin{aligned}
& \iint_{0}^{T} \int_{\Omega}\left(-\bar{\phi}_{m} S_{m}^{\alpha}\left(\bar{p}_{c}\right) \partial_{t} \bar{\varphi}^{\alpha}+\eta_{m}^{\alpha}\left(S_{m}^{\alpha}\left(\bar{p}_{c}\right)\right) \mathbb{K}_{m} \nabla \bar{p}^{\alpha} \cdot \nabla \bar{\varphi}^{\alpha}\right) d \mathbf{x} d t \\
& +\int_{0}^{T} \int_{\Gamma}\left(-\bar{d}_{f} S_{f}^{\alpha}\left(\gamma \bar{p}_{c}\right) \partial_{t} \gamma \bar{\varphi}^{\alpha}+\eta_{f}^{\alpha}\left(S_{f}^{\alpha}\left(\gamma \bar{p}_{c}\right)\right) \frac{\bar{d}_{f}^{3}}{12} \nabla_{\tau} \gamma \bar{p}^{\alpha} \cdot \nabla_{\tau} \gamma \bar{\varphi}^{\alpha}\right) d \sigma(\mathbf{x}) d t \\
& -\int_{\Omega} \bar{\phi}_{m}^{0} S_{m}^{\alpha}\left(\bar{p}_{c}^{0}\right) \bar{\varphi}^{\alpha}(0, \cdot) d \mathbf{x}-\int_{\Gamma} \bar{d}_{f}^{0} S_{f}^{\alpha}\left(\gamma \bar{p}_{c}^{0}\right) \gamma \bar{\varphi}^{\alpha}(0, \cdot) d \sigma(\mathbf{x}) \\
& =\int_{0}^{T} \int_{\Omega} h_{m}^{\alpha} \bar{\varphi}^{\alpha} d \mathbf{x} d t+\int_{0}^{T} \int_{\Gamma} h_{f}^{\alpha} \gamma \bar{\varphi}^{\alpha} d \sigma(\mathbf{x}) d t \\
& \int_{0}^{T} \int_{\Omega}\left(\sigma(\overline{\mathbf{u}}): \mathbb{E}(\overline{\mathbf{v}})-b \bar{p}_{m}^{E} \operatorname{div}(\overline{\mathbf{v}})\right) d \mathbf{x} d t+\int_{0}^{T} \int_{\Gamma} \bar{p}_{f}^{E} \llbracket \overline{\mathbf{v}} \rrbracket d \sigma(\mathbf{x}) d t \\
& =\int_{0}^{T} \int_{\Omega} \mathbf{f} \cdot \overline{\mathbf{v}} d \mathbf{x} d t
\end{aligned}
$$

with $\bar{p}_{c}=\bar{p}^{\mathrm{nw}}-\bar{p}^{\mathrm{w}}, \bar{d}_{f}=-\llbracket \overline{\mathbf{u}} \rrbracket, \bar{\phi}_{m}-\bar{\phi}_{m}^{0}=b \operatorname{div}\left(\overline{\mathbf{u}}-\overline{\mathbf{u}}^{0}\right)+\frac{1}{M}\left(\bar{p}_{m}^{E}-\bar{p}_{m}^{E, 0}\right)$, $\bar{d}_{f}^{0}=-\llbracket \overline{\mathbf{u}}^{0} \rrbracket$, where $\overline{\mathbf{u}}^{0}$ is the solution of (4) without the time integral and using the initial equivalent pressures $\bar{p}_{m}^{E, 0}$ and $\bar{p}_{f}^{E, 0}$ obtained from the initial pressures $\bar{p}_{0}^{\alpha} \in V_{0}, \alpha \in\{\mathrm{nw}, \mathrm{w}\}$.

Remark 1 (Regularity of the displacement field). Notice that $\overline{\mathbf{u}} \in L^{\infty}\left(0, T ; \mathbf{U}_{0}\right)$ implies $\bar{d}_{f}=-\llbracket \overline{\mathbf{u}} \rrbracket \in L^{\infty}\left(0, T ; L^{4}(\Gamma)\right)$. All the integrals above are thus well-defined.

\section{The gradient scheme}

The gradient discretization for the mechanics is defined by the vector space of d.o.f. $X_{\mathcal{D}_{\mathrm{u}}}^{0}$ and 
- a symmetric gradient operator $\mathfrak{D}_{\mathcal{D}_{\mathbf{u}}}: X_{\mathcal{D}_{\mathbf{u}}}^{0} \rightarrow L^{2}\left(\Omega, \mathcal{S}_{d}(\mathbb{R})\right)$,

- a displacement function reconstruction operator $\Pi_{\mathcal{D}_{\mathbf{u}}}: X_{\mathcal{D}_{\mathrm{u}}}^{0} \rightarrow L^{2}(\Omega)^{d}$,

- a normal jump function reconstruction operator $\llbracket \cdot \rrbracket_{\mathcal{D}_{\mathbf{u}}}: X_{\mathcal{D}_{\mathbf{u}}}^{0_{u}} \rightarrow L^{4}(\Gamma)$,

where $\mathcal{S}_{d}(\mathbb{R})$ is the vector space of real symmetric matrices of size $d$. Let us define the divergence operator $\operatorname{div}_{\mathcal{D}_{\mathbf{u}}}(\cdot)=\operatorname{Trace}\left(\mathfrak{C}_{\mathcal{D}_{\mathbf{u}}}(\cdot)\right)$, the stress tensor operator

$$
\sigma_{\mathcal{D}_{\mathbf{u}}}(\mathbf{v})=2 \mu \mathbb{C}_{\mathcal{D}_{\mathbf{u}}}(\mathbf{v})+\lambda \operatorname{div}_{\mathcal{D}_{\mathbf{u}}}(\mathbf{v}) \mathbb{I},
$$

and the fracture width $d_{f, \mathcal{D}_{\mathbf{u}}}=-\llbracket \mathbf{u} \rrbracket_{\mathcal{D}_{\mathbf{u}}}$. It is assumed that $\|\mathbf{v}\|_{\mathcal{D}_{\mathbf{u}}}=\left\|\mathbb{E}_{\mathcal{D}_{\mathbf{u}}}(\mathbf{v})\right\|_{L^{2}(\Omega)}$ is a norm on $X_{\mathcal{D}_{\mathbf{u}}}^{0}$.

The gradient discretization (GD) of the Darcy continuous pressure model is introduced in [1] and defined by the vector space of d.o.f. $X_{\mathcal{D}_{p}}^{0}$ and

- two discrete gradient operators on the matrix and fracture domains

$$
\nabla_{\mathcal{D}_{p}}^{m}: X_{\mathcal{D}_{p}}^{0} \rightarrow L^{\infty}(\Omega)^{d}, \quad \nabla_{\mathcal{D}_{p}}^{f}: X_{\mathcal{D}_{p}}^{0} \rightarrow L^{\infty}(\Gamma)^{d-1} ;
$$

- two function reconstruction operators on the matrix and fracture domains

$$
\Pi_{\mathcal{D}_{p}}^{m}: X_{\mathcal{D}_{p}}^{0} \rightarrow L^{\infty}(\Omega), \quad \Pi_{\mathcal{D}_{p}}^{f}: X_{\mathcal{D}_{p}}^{0} \rightarrow L^{\infty}(\Gamma),
$$

which are piecewise constant [3, Definition 2.12].

A consequence of the piecewise-constant property is that, for any $g: \mathbb{R} \rightarrow \mathbb{R}$ and $v \in X_{\mathcal{D}_{p}}^{0}$, we can define $g(v) \in X_{\mathcal{D}_{p}}^{0}$ component-wise and we have $\Pi_{\mathcal{D}_{p}}^{\rho} g(v)=$ $g\left(\Pi_{\mathcal{D}_{p}}^{\rho} v\right)$ for $\rho \in\{m, f\}$. Fixing a continuous function $d_{0}: \Gamma \rightarrow(0,+\infty)$ with zero limits at the tips of $\Gamma$, the vector space $X_{\mathcal{D}_{p}}^{0}$ is endowed with $\|v\|_{\mathcal{D}_{p}}=$ $\left\|\nabla_{\mathcal{D}_{p}}^{m} v\right\|_{L^{2}(\Omega)^{d}}+\left\|d_{0}^{\frac{3}{2}} \nabla_{\mathcal{D}_{p}}^{f} v\right\|_{L^{2}(\Gamma)^{d-1}}$, assumed to define a norm on $X_{\mathcal{D}_{p}}^{0}$.

This spatial GD is extended into a space-time GD by complementing it with

- a discretisation $0=t_{0}<t_{1}<\cdots<t_{N}=T$ of the time interval $[0, T]$;

- interpolators $P_{\mathcal{D}_{p}}: V_{0} \rightarrow X_{\mathcal{D}_{p}}^{0}$ and $P_{\mathcal{D}_{p}}^{m}: L^{2}(\Omega) \rightarrow X_{\mathcal{D}_{p}}^{0}$ of initial conditions.

The spatial operators are extended into space-time operators as follows. Let $\chi$ represent either $p$ or $\mathbf{u}$. If $w=\left(w_{n}\right)_{n=0}^{N} \in\left(X_{\mathcal{D}_{\chi}}^{0}\right)^{N+1}$, and $\Psi_{\mathcal{D}_{\chi}}$ is a spatial GDM operator, its space-time extension is defined by

$\Psi_{\mathcal{D}_{\chi}}(0, \cdot)=\Psi_{\mathcal{D}_{\chi}}\left(w_{0}\right)$ and, $\forall n \in\{0, \ldots, N-1\}, \forall t \in\left(t_{n}, t_{n+1}\right], \Psi_{\mathcal{D}_{\chi}}(t, \cdot)=\Psi_{\mathcal{D}_{\chi}} w_{n+1}$.

where, for convenience, the same notation is kept for the spatial and space-time operators. We also define the discrete time derivative as follows: for $f:[0, T] \rightarrow L^{1}(\Omega)$ piecewise constant on the time discretisation, with $f_{n}=f_{\mid\left(t_{n-1}, t_{n}\right]}$, and using the same $n$ and $t$ as above, $\delta_{t} f(t)=\frac{f_{n+1}-f_{n}}{t_{n+1}-t_{n}}$.

The gradient scheme for (1) consists in writing the weak formulation (3)-(4) with continuous spaces and operators substituted by their discrete counterparts, after a formal integration by part: find $p^{\alpha} \in\left(X_{\mathcal{D}_{p}}^{0}\right)^{N+1}, \alpha \in\{\mathrm{nw}, \mathrm{w}\}$, and $\mathbf{u} \in\left(X_{\mathcal{D}_{\mathbf{u}}}^{0}\right)^{N+1}$, such that for all $\varphi^{\alpha} \in\left(X_{\mathcal{D}_{p}}^{0}\right)^{N+1}, \mathbf{v} \in\left(X_{\mathcal{D}_{\mathbf{u}}}^{0}\right)^{N+1}$ and $\alpha \in\{\mathrm{nw}, \mathrm{w}\}$, 


$$
\begin{aligned}
& \int_{0}^{T} \int_{\Omega}\left(\delta_{t}\left(\phi_{\mathcal{D}} \Pi_{\mathcal{D}_{p}}^{m} s_{m}^{\alpha}\right) \Pi_{\mathcal{D}_{p}}^{m} \varphi^{\alpha}+\eta_{m}^{\alpha}\left(\Pi_{\mathcal{D}_{p}}^{m} s_{m}^{\alpha}\right) \mathbb{K}_{m} \nabla_{\mathcal{D}_{p}}^{m} p^{\alpha} \cdot \nabla_{\mathcal{D}_{p}}^{m} \varphi^{\alpha}\right) d \mathbf{x} d t \\
& +\int_{0}^{T} \int_{\Gamma} \delta_{t}\left(d_{f, \mathcal{D}_{\mathbf{u}}} \Pi_{\mathcal{D}_{p}}^{f} s_{f}^{\alpha}\right) \Pi_{\mathcal{D}_{p}}^{f} \varphi^{\alpha} d \sigma(\mathbf{x}) \\
& +\int_{0}^{T} \int_{\Gamma}^{T} \eta_{f}^{\alpha}\left(\Pi_{\mathcal{D}_{p}}^{f} s_{f}^{\alpha}\right) \frac{d_{f, \mathcal{D}_{\mathbf{u}}}^{3}}{12} \nabla_{\mathcal{D}_{p}}^{f} p^{\alpha} \cdot \nabla_{\mathcal{D}_{p}}^{f} \varphi^{\alpha} d \mathbf{x} d t \\
& =\int_{0}^{T} \int_{\Omega} h_{m}^{\alpha} \Pi_{\mathcal{D}_{p}}^{m} \varphi^{\alpha} d \mathbf{x} d t+\int_{0}^{T} \int_{\Gamma} h_{f}^{\alpha} \Pi_{\mathcal{D}_{p}}^{f} \varphi^{\alpha} d \sigma(\mathbf{x}) d t, \\
& \int_{0}^{T} \int_{\Omega}\left(\sigma_{\mathcal{D}_{\mathbf{u}}}(\mathbf{u}): \mathbb{C}_{\mathcal{D}_{\mathbf{u}}}(\mathbf{v})-b\left(\Pi_{\mathcal{D}_{p}}^{m} p_{m}^{E}\right) \operatorname{div}_{\mathcal{D}_{\mathbf{u}}}(\mathbf{v})\right) d \mathbf{x} d t \\
& +\int_{0}^{T} \int_{\Gamma}\left(\Pi_{\mathcal{D}_{p}}^{f} p_{f}^{E}\right) \llbracket \mathbf{v} \rrbracket_{\mathcal{D}_{\mathbf{u}}} d \sigma(\mathbf{x}) d t=\int_{0}^{T} \int_{\Omega} \mathbf{f} \cdot \Pi_{\mathcal{D}_{\mathbf{u}}} \mathbf{v} d \mathbf{x} d t
\end{aligned}
$$

with the closure equations

$$
\left\{\begin{array}{l}
p_{c}=p^{\mathrm{nw}}-p^{\mathrm{w}}, \quad s_{m}^{\alpha}=S_{m}^{\alpha}\left(p_{c}\right), \quad s_{f}^{\alpha}=S_{f}^{\alpha}\left(p_{c}\right) \\
p_{m}^{E}=\sum_{\alpha \in\{\mathrm{nw}, \mathrm{w}\}} p^{\alpha} s_{m}^{\alpha}-U_{m}\left(p_{c}\right), \quad p_{f}^{E}=\sum_{\alpha \in\{\mathrm{nw}, \mathrm{w}\}} p^{\alpha} s_{f}^{\alpha}-U_{f}\left(p_{c}\right) \\
\phi_{\mathcal{D}}-\Pi_{\mathcal{D}_{p}}^{m} \bar{\phi}_{m}^{0}=b \operatorname{div}_{\mathcal{D}_{\mathbf{u}}}\left(\mathbf{u}-\mathbf{u}^{0}\right)+\frac{1}{M} \prod_{\mathcal{D}_{p}}^{m}\left(p_{m}^{E}-p_{m}^{E, 0}\right)
\end{array}\right.
$$

The initial conditions are given by $p_{0}^{\alpha}=P_{\mathcal{D}_{p}} \bar{p}_{0}^{\alpha}(\alpha \in\{\mathrm{nw}, \mathrm{w}\}), \phi_{m}^{0}=P_{\mathcal{D}_{p}}^{m} \bar{\phi}^{0}$, and the initial displacement $\mathbf{u}^{0}$ is the solution of $5 \mathrm{~b}$ ) with the equivalent pressures obtained from the initial pressures $\left(p_{0}^{\alpha}\right)_{\alpha \in\{\mathrm{nw}, \mathrm{w}\}}$.

\section{Convergence result}

Let $\left(\mathcal{D}_{p}^{l}\right)_{l \in \mathbb{N}}$ and $\left(\mathcal{D}_{\mathbf{u}}^{l}\right)_{l \in \mathbb{N}}$ be sequences of GDs. We state here the assumptions on these sequences which ensure that the solutions to the corresponding schemes converge. Most of these assumptions are adaptation of classical GDM assumptions [3], except for the chain-rule and cut-off properties, whose role is briefly discussed at the end of the paper; we note that all these assumptions hold for standard discretisations used in porous media flows.

Coercivity, consistency and limit-conformity of $\left(\mathcal{D}_{p}^{l}\right)_{l \in \mathbb{N}}$ : these propreties are omitted since they are similar to those in [1, the only change being the use in the definition of consistency of the $L^{r}$-norm with $r>8$, instead of the $L^{2}$-norm, for the gradient in the fractures, and the use of fracture fluxes $\mathbf{q}_{f}$ compactly supported away from the fracture tips in the definition of the limit-conformity.

Chain rule estimate on $\left(\mathcal{D}_{p}^{l}\right)_{l \in \mathbb{N}}$ : for any Lipschitz-continuous function $F: \mathbb{R} \rightarrow$ $\mathbb{R}$, there is $C_{F} \geqslant 0$ such that, for all $l \in \mathbb{N}$ and $v \in X_{\mathcal{D}_{p}^{l}}^{0},\left\|\nabla_{\mathcal{D}_{p}^{l}}^{m} F(v)\right\|_{L^{2}(\Omega)^{d}} \leqslant$ $C_{F}\left\|\nabla_{\mathcal{D}_{p}^{l}}^{m} v\right\|_{L^{2}(\Omega)^{d}}$.

Cut-off property of $\left(\mathcal{D}_{p}^{l}\right)_{l \in \mathbb{N}}$ : for any compact set $K \subset \Omega \backslash \Gamma$ and $l \in \mathbb{N}$, there exists $\psi^{l} \in X_{\mathcal{D}_{p}^{l}}$ such that, for $l$ large enough and $C \geqslant 0$ not depending on $l: \Pi_{\mathcal{D}_{p}^{l}}^{m} \psi^{l} \geqslant 0$ on $\Omega ; \Pi_{\mathcal{D}_{p}^{l}}^{m} \psi^{l} \geqslant 1$ on $K ;\left\|\nabla_{\mathcal{D}_{p}^{l}}^{m} \psi^{l}\right\|_{L^{2}(\Omega)^{d}} \leqslant C ; \Pi_{\mathcal{D}_{p}^{l}}^{f} \psi^{l}=0$; and $\nabla_{\mathcal{D}_{p}^{l}}^{f} \psi^{l}=0$.

Coercivity of $\left(\mathcal{D}_{\mathbf{u}}^{l}\right)_{l \in \mathbb{N}}$. It holds

$$
\sup _{l \in \mathbb{N}} \max _{\mathbf{v} \in X_{\mathcal{D}_{\mathbf{u}}^{l}}^{0} \backslash\{0\}} \frac{\left\|\Pi_{\mathcal{D}_{\mathbf{u}}^{l}} \mathbf{v}\right\|_{L^{2}(\Omega)^{d}}+\left\|\llbracket \mathbf{v} \rrbracket_{\mathcal{D}_{\mathbf{u}}^{l}}\right\|_{L^{4}(\Gamma)}}{\|\mathbf{v}\|_{\mathcal{D}_{\mathbf{u}}^{l}}}<+\infty
$$


Consistency of $\left(\mathcal{D}_{\mathbf{u}}^{l}\right)_{l \in \mathbb{N}}$. For all $\overline{\mathbf{u}} \in \mathbf{U}_{0}$, it holds $\lim _{l \rightarrow+\infty} \mathcal{S}_{\mathcal{D}_{\mathbf{u}}^{l}}(\overline{\mathbf{u}})=0$ where

$$
\begin{aligned}
\mathcal{S}_{\mathcal{D}_{\mathbf{u}}^{l}}(\overline{\mathbf{u}})=\min _{\mathbf{v} \in X_{\mathcal{D}_{\mathbf{u}}^{\prime}}^{0}}\left[\left\|\mathbb{E}_{\mathcal{D}_{\mathbf{u}}^{l}}(\mathbf{v})-\mathbb{C}(\overline{\mathbf{u}})\right\|_{L^{2}\left(\Omega, \mathcal{S}_{d}(\mathbb{R})\right)}\right. \\
\left.+\left\|\Pi_{\mathcal{D}_{\mathbf{u}}^{l}} \mathbf{v}-\overline{\mathbf{u}}\right\|_{L^{2}(\Omega)^{d}}+\left\|\llbracket \mathbf{v} \rrbracket_{\mathcal{D}_{\mathbf{u}}^{l}}-\llbracket \overline{\mathbf{u}} \rrbracket\right\|_{L^{4}(\Gamma)}\right] .
\end{aligned}
$$

Limit Conformity of $\left(\mathcal{D}_{\mathbf{u}}^{l}\right)_{l \in \mathbb{N}}$. Let $C_{\Gamma}^{\infty}\left(\Omega \backslash \bar{\Gamma}, \mathcal{S}_{d}(\mathbb{R})\right)$ denote the vector space of smooth functions $\sigma(\mathbf{x})$ from $\Omega \backslash \bar{\Gamma}$ to $\mathcal{S}_{d}(\mathbb{R})$ defined as above, and such that $\sigma^{+}(\mathbf{x}) \mathbf{n}^{+}+\sigma^{-}(\mathbf{x}) \mathbf{n}^{-}=\mathbf{0}$ and $\left(\sigma^{+}(\mathbf{x}) \mathbf{n}^{+}\right) \times \mathbf{n}^{+}=\mathbf{0}$ for a.e. $\mathbf{x} \in \Gamma$. For all $\sigma \in C_{\Gamma}^{\infty}\left(\Omega \backslash \bar{\Gamma}, \mathcal{S}_{d}(\mathbb{R})\right)$, it holds $\lim _{l \rightarrow+\infty} \mathcal{W}_{\mathcal{D}_{\mathbf{u}}^{l}}(\sigma)=0$ where

$$
\begin{aligned}
\mathcal{W}_{\mathcal{D}_{\mathbf{u}}^{l}}(\sigma)=\max _{\mathbf{v} \in X_{\mathcal{D}_{\mathbf{u}}^{l}}^{0} \backslash\{0\}} \frac{1}{\|\mathbf{v}\|_{\mathcal{D}_{\mathbf{u}}^{l}}}\left[\int_{\Omega}\left(\sigma: \mathbb{C}_{\mathcal{D}_{\mathbf{u}}^{l}}(\mathbf{v})+\Pi_{\mathcal{D}_{\mathbf{u}}^{l} \mathbf{v}} \operatorname{div}(\sigma)\right) d \mathbf{x}\right. \\
-\int_{\Gamma}\left(\left(\sigma \mathbf{n}^{+}\right) \cdot \mathbf{n}^{+} \llbracket \mathbf{v} \rrbracket_{\mathcal{D}_{\mathbf{u}}^{l}} d \sigma(\mathbf{x})\right] .
\end{aligned}
$$

Compactness of $\left(\mathcal{D}_{\mathbf{u}}^{l}\right)_{l \in \mathbb{N}}$. For any sequence $\left(\mathbf{v}^{l}\right)_{l \in \mathbb{N}}$ with $\mathbf{v}^{l} \in X_{\mathcal{D}_{\mathbf{u}}^{l}}^{0}$ for all $l \in \mathbb{N}$ such that $\sup _{l \in \mathbb{N}}\left\|\mathbf{v}^{l}\right\|_{\mathcal{D}_{\mathbf{u}}^{l}}<+\infty$, the sequences $\left(\Pi_{\mathcal{D}_{\mathbf{u}}^{l}} \mathbf{v}^{l}\right)_{l \in \mathbb{N}}$ and $\left(\llbracket \mathbf{v}^{l} \rrbracket_{\mathcal{D}_{\mathbf{u}}^{l}}\right)_{l \in \mathbb{N}}$ are relatively compact in $L^{2}(\Omega)^{d}$ and in $L^{s}(\Gamma)$ for all $s<4$, respectively.

We can now state the convergence result.

Theorem 1. Let $t_{n}^{l}, n=0, \cdots, N^{l}$ and $l \in \mathbb{N}$, be a sequence of time discretizations such that $\lim _{l \rightarrow+\infty} \max _{n=0, \cdots, N^{l}-1}\left(t_{n+1}^{l}-t_{n}^{l}\right)=0$. Let $0<\phi_{m, \min } \leqslant \phi_{m, \max }<+\infty$ and assume that, for each $l \in \mathbb{N}$, the gradient scheme (5a)-5b has a solution $p_{l}^{\alpha} \in\left(X_{\mathcal{D}_{p}^{l}}^{0}\right)^{N+1}, \alpha \in\{\mathrm{nw}, \mathrm{w}\}, \mathbf{u}^{l} \in\left(X_{\mathcal{D}_{\mathbf{u}}^{l}}^{0}\right)^{N+1}$ such that

(i) $d_{f, \mathcal{D}_{\mathbf{u}}^{l}}(t, \mathbf{x}) \geqslant d_{0}(\mathbf{x})$ for a.e. $(t, \mathbf{x}) \in(0, T) \times \Gamma$,

(ii) $\phi_{m, \text { min }} \leqslant \phi_{\mathcal{D}^{l}}(t, \mathbf{x}) \leqslant \phi_{\text {m,max }}$ for a.e. $(t, \mathbf{x}) \in(0, T) \times \Omega$.

Then, there exist $\bar{p}^{\alpha} \in L^{2}\left(0, T ; V_{0}\right), \alpha \in\{\mathrm{nw}, \mathrm{w}\}$, and $\overline{\mathbf{u}} \in L^{\infty}\left(0, T ; \mathbf{U}_{0}\right)$ solutions of the weak formulation (3)-(4) such that for $\alpha \in\{\mathrm{nw}, \mathrm{w}\}$ and up to a subsequence

$$
\left\{\begin{array}{l}
\Pi_{\mathcal{D}^{l}}^{m} p_{l}^{\alpha} \rightarrow \bar{p}^{\alpha} \text { in } L^{2}\left(0, T ; L^{2}(\Omega)\right), \\
\Pi_{\mathcal{D}_{p}^{l}}^{f^{p}} p_{l}^{\alpha} \rightarrow \gamma \bar{p}^{\alpha} \text { in } L^{2}\left(0, T ; L^{2}(\Gamma)\right), \\
\Pi_{\mathcal{D}_{\mathbf{u}}^{l} \mathbf{u}} \mathbf{u}^{l} \mathbf{\mathbf { u }} \text { in } L^{\infty}\left(0, T ; L^{2}(\Omega)^{d}\right) \text { weak } \star \\
d_{f, \mathcal{D}_{\mathbf{u}}^{l}} \rightarrow \bar{d}_{f} \text { in } L^{\infty}\left(0, T ; L^{p}(\Gamma)\right) \text { for } 2 \leqslant p<4, \\
\phi_{\mathcal{D}^{l}} \rightarrow \bar{\phi}_{m} \text { in } L^{\infty}\left(0, T ; L^{2}(\Omega)\right) \text { weak }{ }^{2} \\
\Pi_{D_{p}^{l}}^{m} S_{m}^{\alpha}\left(p_{c}^{l}\right) \rightarrow S_{m}^{\alpha}\left(\bar{p}_{c}\right) \text { in } L^{2}\left(0, T ; L^{2}(\Omega)\right), \\
\Pi_{D_{p}^{l}}^{f^{l}} S_{f}^{\alpha}\left(p_{c}^{l}\right) \rightarrow S_{f}^{\alpha}\left(\gamma \bar{p}_{c}\right) \text { in } L^{2}\left(0, T ; L^{2}(\Gamma)\right) .
\end{array}\right.
$$

The proof of Theorem 1 hinges on the following steps:

- Inferring energy estimates by using suitable test functions;

- Obtaining weak estimates on time derivatives;

- Using the discontinuous Ascoli-Arzelà compactness theorem [3, Theorem C.11] to prove convergences;

- Identifying the limit fields.

We report here the energy estimate satisfied by the discrete unknowns. For brevity, let $\delta t^{\left(n+\frac{1}{2}\right)}=t_{n+1}-t_{n}$ and $\hat{v}(t)=v\left(t-\delta t^{\left(n+\frac{1}{2}\right)}\right) \forall t \in\left(t_{n}, t_{n+1}\right]$ for a piecewise constant scalar or vector function $v$ on $[0, T]$. Upon choosing $\varphi^{\alpha}=p^{\alpha}$ in (5a) and $\mathbf{v}=\delta_{t} \mathbf{u}(t)$ in (5b), using the fact that $\delta_{t}(u v)(t)=\hat{u}(t) \delta_{t} v(t)+v(t) \delta_{t} u(t)$, summing the corresponding equations, and using the closure equations (5c) along 
with the assumptions we made on the data, we obtain the following estimate for the solutions of (5): there is a real number $C>0$ depending on the data such that

$$
\begin{aligned}
& \int_{0}^{T} \int_{\Omega} \delta_{t}\left(\phi_{\mathcal{D}} U_{m}\left(\Pi_{\mathcal{D}_{p}}^{m} p_{c}\right)\right) d \mathbf{x} d t+\int_{0}^{T} \int_{\Gamma} \delta_{t}\left(d_{f, \mathcal{D}_{\mathbf{u}}} U_{f}\left(\Pi_{\mathcal{D}_{p}}^{f} p_{c}\right)\right) d \mathbf{x} d t \\
& +\int_{0}^{T} \int_{\Omega} \delta_{t}\left(\frac{1}{2}\left(\sigma_{\mathcal{D}_{\mathbf{u}}}(\mathbf{u}): \mathbb{D}_{\mathcal{D}_{\mathbf{u}}}(\mathbf{u})\right)+\frac{1}{2 M}\left(\Pi_{\mathcal{D}_{p}}^{m} p_{m}^{E}\right)^{2}\right) d \mathbf{x} d t \\
& +\sum_{\alpha \in\{\mathrm{w}, \mathrm{nw}\}} \int_{0}^{T} \int_{\Omega}\left|\nabla_{\mathcal{D}_{p}}^{m} p^{\alpha}\right|^{2} d \mathbf{x} d t+\sum_{\alpha \in\{\mathrm{w}, \mathrm{nw}\}} \int_{0}^{T} \int_{\Gamma} d_{f, \mathcal{D}_{\mathbf{u}}}^{3}\left|\nabla_{\mathcal{D}_{p}}^{f} p^{\alpha}\right|^{2} d \mathbf{x} d t \\
& \leqslant C\left(\int_{0}^{T} \int_{\Omega} \mathbf{f} \cdot \delta_{t} \Pi_{\mathcal{D}_{\mathbf{u}}} \mathbf{u} d \mathbf{x} d t+\sum_{\alpha \in\{\mathrm{w}, \mathrm{nw}\}} \int_{0}^{T} \int_{\Omega} h_{m}^{\alpha} \Pi_{\mathcal{D}_{p}}^{m} p^{\alpha} d \mathbf{x} d t\right. \\
& \left.\quad+\sum_{\alpha \in\{\mathrm{w}, \mathrm{nw}\}} \int_{0}^{T} \int_{\Gamma} h_{f}^{\alpha} \Pi_{\mathcal{D}_{p}}^{f} p^{\alpha} d \mathbf{x} d t\right) .
\end{aligned}
$$

The right-hand side of this inequality is made of positive terms (up to initial conditions, that appear in the telescopic sums corresponding to the first three terms), with enough quadratic growth in the unknowns to compensate the linear dependency of the right-hand side on these unknowns.

The chain-rule estimates and cut-off properties of $\left(\mathcal{D}_{p}^{l}\right)_{l \in \mathbb{N}}$ are used to prove estimates on the time-translates of $\Pi_{D_{p}^{l}}^{m} S_{m}^{\alpha}\left(p_{c}^{l}\right)$ (which are crucial in establishing the strong convergence of this quantity). These estimates require to separate the matrix and fracture components (hence the need for using cut-off test functions in the scheme), and is based on a dual estimate that requires to use $S_{m}^{\alpha}\left(p_{c}^{l}\right)$ as a test function and estimate its gradient (which follows from gradient estimates on $p_{c}^{l}$ and the chain-rule estimates).

Acknowledgements We are grateful to Andra and to the Australian Research Council's Discovery Projects (project DP170100605) funding scheme for partially supporting this work.

\section{References}

1. Brenner, K., Groza, M., Guichard, C., Lebeau, G., Masson, R.: Gradient discretization of hybrid-dimensional darcy flows in fractured porous media. Numerische Mathematik 134(3), 569-609 (2016)

2. Coussy, O.: Poromechanics. John Wiley \& Sons (2004)

3. Droniou, J., Eymard, R., Gallouët, T., Guichard, C., Herbin, R.: The gradient discretisation method, Mathematics \& Applications, vol. 82. Springer (2018). DOI 10.1007/978-3-319-79042-8

4. Girault, V., Wheeler, M., Ganis, B., Mear, M.: A lubrication fracture model in a poroelastic medium. Mathematical Models and Methods in Applied Sciences 25, 4 (2015) 